\title{
La captivité des combattants irlandais de la Première Guerre mondiale : propagande de guerre, transferts de loyauté et résistances
}

\author{
EMMANUEL DESTENAY
}

Les historiens de la Première Guerre mondiale se sont longtemps surtout intéressés aux combats, et ont de ce fait souvent délaissé les expériences de guerre des prisonniers. L'internement ne leur apparaissait pas «comme un facteur valorisant de l'univers combattant ${ }^{1} \gg$. Or, depuis quelques années, une plus grande attention est portée à la manière dont ont été traités ces hommes ${ }^{2}$. Ces recherches envisagent généralement les combattants des armées impériales comme des groupes homogènes alors que leurs conditions d'internement diffèrent selon les groupes ethniques auxquels ils appartiennent. Ainsi des soldats venus des colonies françaises sont regroupés par le gouvernement allemand, qui tente de gagner leur confiance et de les forcer à renier leur uniforme ${ }^{3}$. De même, les combattants irlandais de l'armée britannique connaissent un traitement différent de celui de leurs frères d'armes. Les études consacrées à la captivité des combattants britanniques de la Première Guerre mondiale envisagent cette armée comme une entité homogène, négligeant ainsi les particularités des conditions d'internement des captifs irlandais ${ }^{4}$. Pourtant, le gouvernement allemand sépare ces derniers des autres prisonniers britanniques quelques mois après le début des hostilités et les regroupe dans un seul et même camp.

L'étude du sort des quelque 2000 captifs irlandais regroupés dans le camp de Limburg an der Lahn ${ }^{5}$ permet d'ouvrir un nouveau chapitre dans l'historiographie des violences infligées aux prisonniers de guerre. En effet, un corpus documentaire disponible aux Archives Nationales de Londres, jusqu'alors très peu sollicité, permet d'étudier les conditions d'internement de ces hommes. La Commission Gouvernementale sur le Traitement des Prisonniers de Guerre Britanniques ${ }^{6}$, établie en 1915 par le Ministère des Affaires étrangères britannique, consigne les témoignages des prisonniers évadés ou rapatriés des camps allemands. Le gouvernement britannique peut ainsi établir dès 1916 un Rapport sur les visites

\footnotetext{
1 Odon Abbal, «Historiographie de la captivité », in Jean-Charles Jauffret, Jules Maurin (dir.), La Grande Guerre 1914-1918 : 80 ans d'historiographie et de représentations : colloque international, Montpellier, 20-21 novembre 1998, Montpellier, Université Paul Valery, 2002, p. 164.

2 Jules Maurin, Armée, guerre et société : soldats languedociens, 1889-1919, Paris, Publications de la Sorbonne, 1982, 750 p. François Cochet, Soldats sans armes. La captivité de guerre, une approche culturelle, Bruxelles, Éditions Bruylant, 1998, 464 p ; Odon Abbal, Soldats oubliés. Les prisonniers de guerre, Bez-et-Esparon, Études et Communication Éditions, 2001, 272 p ; Jean-Claude Auriol, Les Barbelés des bannis. La tragédie des prisonniers de guerre français en Allemagne pendant la Grande Guerre, Paris, Éditions Tirésias, 2003, 284 p ; Frédéric Médard, Les prisonniers en 1914-1918. Acteurs méconnus de la Grande Guerre, Paris, Éditions Soteca, 2010, 350 p.

${ }^{3}$ Annette Becker, Oubliés de la Grande Guerre, humanitaires et culture de guerre : populations occupées, déportés civils, prisonniers de guerre, Paris, Noêsis, 1998, p. 293.

${ }^{4}$ Heather Jones, Violence against Prisoners of War in the First World War: Britain, France and Germany, 19141920, Cambridge, Cambridge University Press, 2011, 468 p.

5 Archives Nationales de Londres (désormais TNA), WO 141/49, «Rapport sur le camp de prisonniers de Limburg et sur la tentative de formation d'une brigade irlandaise, $1921 »$, p. 9.

6 Ibidem, WO 161/98-136, «Commission sur le traitement des prisonniers britanniques dans les camps allemands, rapports individuels de prisonniers. »
} 
de Sir Casement au camp de Limburg ${ }^{7}$ et quelques années après, en 1921, un Rapport sur les Prisonniers de guerre de Limburg et sur la Tentative de Formation d'une Brigade irlandaise ${ }^{8}$. La comparaison de ces rapports officiels avec la correspondance inédite disponible à la Bibliothèque nationale de Dublin traitant de la levée d'une brigade irlandaise pendant le conflit ${ }^{9}$ permet de donner un éclairage nouveau sur les variations des traitements infligés aux prisonniers irlandais mais aussi de comprendre à quel point la situation politique irlandaise à la veille de la Première Guerre mondiale façonne le regard que l'ennemi allemand porte sur les troupes irlandaises ${ }^{10}$. Au-delà même d'une étude strictement limitée aux conditions d'internement des prisonniers irlandais, il s'agit également de comprendre que les velléités d'indépendance irlandaise nourrissent les desseins allemands visant à déstabiliser la GrandeBretagne en temps de guerre.

\section{REGROUPER, DEPLACER ET «SEDUIRE » LES CAPTIFS IRLANDAIS}

En août 1914, l'armée britannique compte 20000 soldats ainsi que 30000 réservistes irlandais prêts à servir en cas de conflit ${ }^{11}$. Les bataillons irlandais du Corps Expéditionnaire Britannique (BEF) qui débarquent en France dès les premiers mois du conflit sont tous issus d'une armée de métier. Les quelque 134202 engagés volontaires irlandais qui revêtent l'uniforme britannique entre août 1914 et novembre 1918 ne participent d'ailleurs pas aux premières batailles ${ }^{12}$. Les trois divisions irlandaises levées pour l'effort de guerre essuient leurs premiers combats plusieurs mois après le début des hostilités. La $10^{\text {ème }}$ division quitte la Grande-Bretagne en juin 1915 pour les Dardanelles ${ }^{13}$, tandis que les $36^{\text {ème }}$ et $16^{\text {ème }}$ divisions débarquent respectivement en France en octobre et décembre $1915^{14}$.

En août 1914, 70000 combattants du Corps Expéditionnaire Britannique quittent la Grande-Bretagne pour la France. À son arrivée sur le continent, le BEF compte dans ses rangs quelque 15000 officiers et soldats irlandais ${ }^{15}$. Quatre régiments de cavalerie et neuf régiments d'infanterie irlandais combattent sous les ordres de Sir French, commandant-en-chef du Corps Expéditionnaire britannique ${ }^{16}$. Les premières grandes offensives menées par les troupes allemandes obligent très vite les divisions britanniques à battre en retraite. Au fur et à mesure que celles-ci se retirent, les Allemands capturent un nombre important de prisonniers. Entre août et décembre 1914 les Allemands regroupent les captifs britanniques dans plusieurs camps. Les captifs irlandais du Corps Expéditionnaire britannique parcourent avec leurs camarades britanniques les territoires occupés du Nord et de l'Est de la France.

\footnotetext{
${ }^{7}$ TNA, WO 141/15, « Rapport sur les visites de Sir Casement au camp de Limburg, 1916 ».

${ }^{8}$ Ibidem, WO 141/49, « Rapport sur le camp de prisonniers de Limburg et sur la tentative de formation d'une brigade irlandaise, $1921 »$.

${ }^{9}$ Librairie Nationale d'Irlande (désormais NLI), MS 13.085/7a, « Mémorandum de Roger Casement au sujet de la Brigade irlandaise », 7 mai 1915.

${ }^{10}$ Jérôme aan de Wiel, The Irish Factor, 1899-1919: Ireland's Strategic and Diplomatic Importance for Foreign Powers, Dublin, Irish Academic Press, 2010, 488 p.

${ }^{11}$ Archives Nationales de Londres (désormais TNA), WO 162/27, Cd. 8168, « Rapport sur le recrutement en Irlande, $1916 »$, p. 2.

${ }^{12}$ Statistics of Military Effort of the British Empire during the Great War, Londres, HMSO, 1922, p. 363. À cette époque, la population totale de l'île est estimée à 4374500.

${ }^{13}$ Bryan Cooper, The 10 ${ }^{\text {th }}$ (Irish) Division at Gallipoli (1918), Dublin, Irish Academic Press, 1995, p. 32.

${ }^{14}$ Cyril Falls, The History of the $36^{\text {th }}$ (Ulster) Division, Londres, Constable, 1922, 359 p; Terence Denman, Ireland's Unknown Soldiers: the $16^{\text {th }}$ Irish Division in the Great War, Dublin, Irish Academic Press, 1992,209 p. ${ }^{15}$ Tom Johnstone, Orange, Green and Khaki: the story of the Irish regiments in the Great war, 1914-18, Dublin, Gill and Macmillan, 1992, p. 6.

${ }^{16}$ Henry Harris, The Irish Regiments in the First World War, Cork, Mercier Press, 1968, p. 33; Tom Johnstone, Orange, Green and Khaki, op. cit., p.5.
} 
Après sa capture à Étreux le 27 août 1914, Michael Healey passe ses premières semaines d'enfermement à Sennelager, en Westphalie, avec ses camarades du $2^{\text {ème }}$ bataillon des Royal Munster Fusiliers ${ }^{17}$. Après plusieurs heures de combat qui permirent de retarder l'avancée des armées allemandes et de battre en retraite, le lieutenant du bataillon, seul officier encore en vie, ordonna à ses hommes de se rendre ${ }^{18}$. Le 26 août, lors de la bataille de Grand-Fayt, une cinquantaine de combattants du $2^{\text {ème }}$ bataillon des Connaught Rangers tombent aux mains des Allemands ${ }^{19}$. Les rescapés parviennent à battre en retraite et à se retrancher vers Maroilles, pour finalement, à l'aube du 27 août, se voir surpris par l'ennemi et capturés. Ce sont 180 combattants que les Allemands parviennent ainsi à prendre sans échange de tirs ${ }^{20}$. Le 29 août 1914, sont capturés le soldat de première classe Jospeh Mahoney et d'autres combattants du $2^{\text {ème }}$ bataillon des Royal Irish Rifles à Mons ${ }^{21}$. À quelques kilomètres de là, près d'Avesnes, William Fagan, du $2^{\text {ème }}$ bataillon des Connaught Rangers, tombe aux mains de l'ennemi ${ }^{22}$. Ryan Robert, capturé le 20 octobre 1914 à La Basée, près de Neuve-Chapelle, est interné au camp de Hameln ${ }^{23}$. Michael O'Connor, lui aussi capturé le 20 octobre 1914 à La Basée, est transporté à l'hôpital de Darmstadt, où il subit une amputation $\mathrm{du}$ bras droit. Quelques jours après sa convalescence, ce soldat du $2^{\text {eme }}$ bataillon des Royal Irish Regiment quitte l'hôpital pour le camp de Darmstadt, où il reste une quinzaine de jours avant de partir, lui aussi, pour le camp de Limburg an der Lahn ${ }^{24}$. Les soldats irlandais du Corps Expéditionnaire britannique vivent ces premières semaines de captivité de la même manière que leurs frères d'arme britanniques. À en croire les témoignages recueillis auprès des soldats, il ne semble pas y avoir alors de traitement spécifique envers les prisonniers irlandais.

À partir de décembre 1914 pourtant, l'État-major allemand opère une distinction entre les prisonniers irlandais et les autres combattants britanniques et regroupe désormais les captifs de nationalité irlandaise dans le camp de Limburg, à une cinquantaine de kilomètres au nord-ouest de Francfort-sur-le-Main, où arrivent en provenance de Sennelager environ1500 prisonniers irlandais le 13 décembre $1914^{25}$. John Holden du Royal Irish Regiment reste quatre mois dans ce camp avant de partir en décembre 1914 pour celui de Limburg ${ }^{26}$. Le soldat William Arthur, originaire de la région de Cheshire, en Angleterre, capturé avec son unité des Royal Munster Fusiliers à Étreux le 24 août 1914, note que les Allemands regroupent tous les combattants de nationalité irlandaise dans «des cabanons séparés dans le $\operatorname{camp}^{27} \gg$. Johnn Beattie, $1^{\text {er }}$ bataillon des Gordon Highlanders, se souvient lui aussi que des

\footnotetext{
17 TNA, WO/161/98/155, «Commission sur le traitement des prisonniers britanniques dans les camps allemands, rapports individuels de prisonniers. »

${ }^{18}$ Henry Harris, The Irish Regiments, op. cit., p. 34.

19 Tom Johnstone, Orange, Green and Khaki, op. cit., p. 22.

${ }^{20}$ Colonel H.N. Thompson, 'An account of my capture and experiences in Germany', Journal of the Royal Army Medical Corps, 1915, Vol. 24 (2), pp. 121-136.

${ }^{21}$ TNA, WO 161/98/245, «Commission sur le traitement des prisonniers britanniques dans les camps allemands, rapports individuels de prisonniers. »

${ }_{22}$ Ibidem, WO 161/98/454, «Commission sur le traitement des prisonniers britanniques dans les camps allemands, rapports individuels de prisonniers. »

23 Ibidem, Ministère de la Guerre, WO 161/100/1131, «Commission sur le traitement des prisonniers britanniques dans les camps allemands, rapports individuels de prisonniers. »

${ }^{24}$ Ibidem, WO 141/9, «Formation d'une brigade irlandaise parmi les prisonniers de guerre en Allemagne », témoignage du caporal Michael O’Connor, 12 novembre 1915.

${ }^{25}$ NLI, MS 13.085/7a, « Mémorandum de Roger Casement au sujet de la Brigade irlandaise », 7 mai 1915.

${ }^{26}$ TNA, WO 161/98/135, «Commission sur le traitement des prisonniers britanniques dans les camps allemands, rapports individuels de prisonniers. »

27 Ibidem, WO 161/98/259, «Commission sur le traitement des prisonniers britanniques dans les camps allemands, rapports individuels de prisonniers », témoignage de William Arthur.
} 
prisonniers irlandais quittant le camp pour un endroit « isolé des autres soldats britanniques et alliés $^{28} »$.

William Purcell, originaire de Ballangary, dans le comté de Tipperary, capturé par les Allemands lors de la retraite de Mons le 23 août 1914, s'entretient avec un interprète allemand qui leur expose les raisons de leur départ du camp de Parchim ${ }^{29}$. Le soldat Ryan Robert et ses frères d'armes apprennent aussi l'imminence de leur départ vers un autre camp « où il y aurait beaucoup à manger ${ }^{30}$ ». Les autres prisonniers britanniques envient très vite le sort de leurs camarades si bien que certains captifs anglais, écossais ou gallois se réfugient derrière une fausse identité irlandaise afin d'échapper à la faim et d'obtenir de meilleures conditions d'internement ${ }^{31}$. Alors que des témoignages certifient bien que des Feldwebel allemands séparent naturellement les captifs irlandais du reste des prisonniers ${ }^{32}$, d'autres récits soutiennent que les gardiens allemands ne parviennent pas toujours à décrypter les insignes régimentaires et se fondent uniquement sur la parole des hommes.

Ce regroupement des prisonniers irlandais révèle bien une intention de la part de l'État-major allemand. Rappelons qu'à la veille du Premier conflit mondial, la société irlandaise se trouve divisée entre les partisans d'un État autonome et les unionistes, majoritairement implantés dans le nord du pays. Alors que l'île fait toujours partie du Royaume-Uni, le pays se déchire entre les partisans du Home Rule, projet constitutionnel envisageant de donner une relative autonomie à l'Irlande tout en la conservant sous la tutelle de la Grande-Bretagne, et les unionistes, fidèles partisans de la Couronne britannique, opposés à l'établissement d'un Parlement irlandais auquel la province d'Ulster devrait prêter allégeance. À la veille de la Première Guerre mondiale, la société irlandaise s'arme en vue d'une guerre civile. Le vote du Home Rule par le Parlement britannique en janvier 1913 exacerbe les tensions entre les partisans et adversaires du maintien de l'Irlande au sein du Royaume-Uni. Les forces insurrectionnelles nationalistes et unionistes de l'île se préparent à un affrontement armé.

La déclaration de guerre de l'Angleterre à l'Allemagne, le 4 août 1914, et la décision des autorités britanniques de reporter la mise en place du Home Rule à la fin des hostilités surprennent le peuple irlandais et suspendent immédiatement toute possibilité de conflit armé en Irlande. Alors que les unionistes appuient de manière inconditionnelle la Grande-Bretagne et que les députés nationalistes soutiennent le gouvernement britannique dans sa lutte contre l'Allemagne, quelques nationalistes particulièrement intransigeants voient le conflit comme l'occasion de se soulever contre la présence britannique et de déclarer l'indépendance de l'île.

Le 9 septembre 1914, quelques semaines seulement après l'éclatement des hostilités en Europe, des indépendantistes irlandais partisans d'un soulèvement national se réunissent pour discuter d'une possible aide matérielle allemande. Ces rebelles envisagent une intervention allemande sur le territoire irlandais pour appuyer les forces d'autodéfense irlandaises contre l'armée britannique. Profitant de la neutralité des États-Unis, Roger Casement, ancien consul britannique converti à la cause nationaliste irlandaise et figure emblématique de la lutte contre l'impérialisme ${ }^{33}$, rencontre, en septembre 1914,

\footnotetext{
${ }^{28}$ TNA, WO 161/101, Index 3/751, «Commission sur le traitement des prisonniers britanniques dans les camps allemands : rapports individuels de prisonniers. »

${ }^{29}$ Ibidem, WO 161/99/997, «Commission sur le traitement des prisonniers britanniques dans les camps allemands, rapports individuels de prisonniers. »

${ }^{30}$ Ibidem, WO 161/100/1131, «Commission sur le traitement des prisonniers britanniques dans les camps allemands, rapports individuels de prisonniers. »

31 Ibidem, WO 161/98/1998, «Commission sur le traitement des prisonniers britanniques dans les camps allemands, rapports individuels de prisonniers. »

${ }^{32}$ Ibidem, WO 141/49, « Rapport sur le camp de prisonniers de Limburg et sur la tentative de formation d'une brigade irlandaise, $1921 »$, p. 1.

${ }^{33}$ Brian Inglis, Roger Casement, Londres, Hodder \& Stoughton Limited, 1973, 448 p.
} 
l'ambassadeur d'Allemagne à Washington et son attaché militaire, le capitaine Franz von Papen $^{34}$. Le 2 novembre, Arthur Zimmerman, sous-secrétaire d'État aux Affaires étrangères, le reçoit à Berlin pour conclure un accord. Le Britannique cèle un pacte avec les autorités allemandes qui acceptent de livrer 200000 fusils, dix canons et cinq millions de munitions pour une rébellion nationale et disent vouloir soutenir la cause irlandaise dans l'éventualité d'une victoire de l'Allemagne sur les Alliés ${ }^{35}$.

Avant le déclenchement des hostilités en Europe, Berlin s'intéressait de près à la situation politique irlandaise, espérant jouer sur les velléités d'indépendance pour « déstabiliser [les puissances européennes] avec l'aide de nationalités allogènes ${ }^{36}$ » en leur apportant une aide matérielle. Une telle diversion avantagerait les Allemands. Une rébellion en Irlande affaiblirait considérablement la Grande-Bretagne, la forçant à « conserver des troupes importantes pour maintenir la tranquillité en Irlande $^{37}$ ». L'intérêt des autorités allemandes pour l'Irlande s'inscrit ici dans une vaste entreprise de déstabilisation des puissances de l'Entente. Quelques semaines après le début des hostilités, une délégation allemande avait d'ailleurs rencontré des indépendantistes finlandais à Stockholm, leur promettant une aide matérielle contre l'Empire russe ${ }^{38}$. En ce sens, l'Irlande ne représente pas un cas singulier. Cependant, les accords conclus entre les autorités allemandes et le diplomate britannique, fervent défenseur d'une indépendance irlandaise, induisaient une intervention militaire et la levée d'une brigade irlandaise composée exclusivement de prisonniers irlandais. Roger Casement et Arthur Zimmerman espéraient ainsi « recruter 200 à 300 hommes parmi les 2200 soldats irlandais catholiques internés à Limburg ${ }^{39}$ ».

L'article 2 du traité stipulait l'objectif de la brigade : «combattre pour la cause irlandaise $^{40} »$. Nourris, équipés et habillés par le gouvernement allemand (article 4), les hommes seraient encadrés et entraînés par des officiers allemands (article 5) ${ }^{41}$. Dans l'éventualité d'une victoire navale face à l'Angleterre, l'armée de Guillaume II acheminerait la brigade vers les côtes irlandaises (article 6) afin que les anciens prisonniers de guerre puissent participer à la libération de leur nation. Combattre auprès des troupes de Guillaume II ne signifiait pas forcément déployer la brigade sur le territoire irlandais. Les «transfuges » pourraient être amenés à prêter main forte aux autres puissances de l'Alliance. L'article $7 \mathrm{du}$ traité évoquait même la possibilité «d'envoyer les combattants irlandais sur le front oriental afin de prêter assistance aux forces de l'Empire ottoman ${ }^{42} »$ pour libérer l'Égypte. L'article 8 précisait que des contacts avec l'Autriche-Hongrie seraient pris afin que l'Empire achemine les combattants irlandais vers Constantinople. La brigade irlandaise devait ainsi se tenir à la disposition des Allemands mais aussi des Ottomans ${ }^{43}$. Il semblait plus envisageable

\footnotetext{
${ }^{34}$ Charles Tansill, America and the Fight for Irish Freedom 1866-1922, New York, The Devin-Adair Co., 1957, p. 178.

${ }^{35}$ Idem.

${ }^{36}$ Maurice Carrez, « Première Guerre mondiale et identité nationale en Finlande », in Rémy Cazals, André Loez (dir.), 1914-1918, Identités troublées. Les appartenances sociales et nationales à l'épreuve de la guerre, Toulouse, Éditions Privat, 2011, p. 302.

37 Ministère des Affaires étrangères, la Courneuve, 1CPCOM545, Cabinet du Ministre, « Correspondance politique et commerciale, Guerre 1914-1918, Irlande », rapport du colonel de la Panouse, attaché militaire à l'Ambassade de la République française à Londres, à Monsieur le Ministre de la Guerre, 3 mai 1916.

${ }^{38}$ Maurice Carrez, « Première Guerre mondiale et identité nationale en Finlande », op. cit., pp. 299-312.

${ }^{39}$ NLI, MS 13.085/7a, « Mémorandum de Roger Casement au sujet de la Brigade irlandaise », 7 mai 1915.

${ }^{40}$ Ibidem, MS 13.085/7, «Accords et clauses du 28 décembre 1914 pour la levée de la brigade irlandaise », article 2 .

${ }^{41}$ Ibidem, MS 13.085/17, «Correspondance de Roger Casement », lettre de H. Haln à Roger Casement, 24 octobre 1915 , p. 1.

${ }^{42}$ Ibidem, MS 13.085/2, « Mémorandum concernant les objectifs de la brigade irlandaise », 1 décembre 1915.

${ }^{43}$ Ibidem, MS 13.085/7, "Accords et clauses du 28 décembre 1914 pour la levée de la brigade irlandaise », article 2 .
} 
d'acheminer la brigade irlandaise dans les Balkans, en Turquie ou en Égypte plutôt qu'en Irlande dans la mesure où cela supposait au préalable la défaite de la flotte britannique.

\section{SURPRISE, INCOMPREHENSION ET PROPAGANDE DE GUERRE}

À leur arrivée au camp de Limburg, un sentiment d'incompréhension s'empare des prisonniers irlandais. William Dooly arrive de Döberitz en décembre 1914 et remarque avec surprise qu'une quantité importante de pain est distribuée. Ryan Robert, arrivé le $1^{\text {er }}$ décembre 1914 avec 280 autres combattants, se souvient qu'à leur arrivée au camp, les gardes leur offrent une double ration de pain «avec un bon souper ${ }^{44}$ ». Les captifs se réjouissent de recevoir des pommes de terre, du riz, et du porc ${ }^{45}$. Durant les trois semaines qui suivent, les combattants irlandais s'estiment bien traités. Il leur est même permis de fumer à l'extérieur des cabanons ${ }^{46}$. Les captifs peuvent également consulter des journaux étrangers lors de leur séjour à Limburg ${ }^{47}$. Les conditions d'internement paraissent privilégiées : de «bons baraquements en bois, bien ventilés, confortables [avec] des tréteaux en bois et suffisamment de couvertures ${ }^{48}$ », «beaucoup de pain $^{49}$ ». La faim épargne les captifs irlandais qui, nouvellement acheminés vers le camp de Limburg, reçoivent une nourriture abondante.

Les prisonniers ignorent toujours les raisons d'une telle clémence. Leur étonnement s'accroît lorsqu'ils reçoivent la visite de Roger Casement quelques jours après leur arrivée. C'est ébahis que les captifs accueillent l'ancien diplomate britannique qui distribue des pamphlets pro-allemands et entame ensuite un discours par: "Irlandais, voici venue pour vous l'occasion de vous battre pour l'Irlande $!^{50}{ }^{\prime}$ Le 6 décembre, lors de la messe dominicale, le Britannique harangue les prisonniers. Insistant sur la nécessité de lever une brigade irlandaise et de rallier la cause des Allemands, Sir Casement exhorte les soldats à profiter du conflit pour déclarer l'indépendance de l'Irlande et combattre aux côtés des Allemands contre l'Angleterre ${ }^{51}$.

De nombreux témoignages confirment la présence de Roger Casement au camp de Limburg. Ryan Robert confie que trois semaines après son arrivée à Limburg en décembre 1914, les prisonniers reçoivent la visite de Sir Casement ${ }^{52}$. Thomas Fahey, du Royal Army Medical Corps, originaire de Carrick-on-Suir, se souvient lui aussi de l'intervention du diplomate en faveur de la victoire allemande. Écouter le discours de l'ancien diplomate s'apparente à un supplice pour les prisonniers qui manifestent leur mécontentement, forçant l'émissaire des républicains irlandais à quitter le camp sous les huées. Certains soldats insultent ouvertement l'orateur tandis que d'autres, à l'instar du sergent Murphy, vont même jusqu'à lui jeter une poignée de boue au visage ${ }^{53}$. Les captifs répondent avec acrimonie à Sir

\footnotetext{
44 TNA, WO 161/100/1131, «Commission sur le traitement des prisonniers britanniques dans les camps allemands, rapports individuels de prisonniers. »

${ }^{45}$ Ibidem., WO 141/49, « Rapport sur le camp de prisonniers de Limburg et sur la tentative de formation d'une brigade irlandaise, $1921 »$, p. 12.

46 Ibidem, WO 161/98/135, «Commission sur le traitement des prisonniers britanniques dans les camps allemands, rapports individuels de prisonniers. »

${ }^{47}$ Ibidem, WO 161/98/135-136, «Commission sur le traitement des prisonniers britanniques dans les camps allemands, rapports individuels de prisonniers. »

${ }^{48}$ NLI, MS 13.085/7a, « Mémorandum de Roger Casement au sujet de la Brigade irlandaise », 7 mai 1915.

49 TNA, WO 161/98/168, «Commission sur le traitement des prisonniers britanniques dans les camps allemands, rapports individuels de prisonniers ».

${ }^{50}$ The Irish Soldier, ${ }^{\mathrm{er}}$ septembre 1918, p. 6.

${ }^{51}$ Idem.

52 TNA, Ministère de la Guerre, WO 161/100/1131, «Commission sur le traitement des prisonniers britanniques dans les camps allemands, rapports individuels de prisonniers ».

${ }^{53}$ The Irish Soldier, 16 octobre 1918, p. 2.
} 
Casement. «Foutez-le dehors !» s'emporte un soldat ${ }^{54}$. S'indigner, c'est non seulement continuer la guerre mais rappeler sa loyauté envers la Couronne britannique. Seuls deux captifs du $2^{\text {eme }}$ bataillon du Royal Irish Regiment, le soldat Michael Keogh et le caporal Timothy Quinslik, visiblement séduits par les promesses, acceptent de prêter allégeance à Guillaume II et quittent le camp ${ }^{55}$.

La résistance collective des captifs irlandais est suivie d'une quinzaine de jours de punition et d'une réduction de leur ration quotidienne de pain qui passe de 750 à 300 grammes. Quatre-vingts prisonniers irlandais «préfèrent mourir de faim plutôt que de revêtir l'uniforme allemand ${ }^{56} »$. Quinze jours plus tard, leurs rations sont diminuées de moitié et les prisonniers sont mis au travail. Au fur et à mesure que les semaines de captivité passent, les conditions «se dégradent ${ }^{57}$ ». Le refus de collaboration avec l'Empire allemand se répercute sur leurs conditions d'internement. Les hommes comprennent très vite que les privations visent à les briser et à les inciter à renier l'uniforme britannique. Les Allemands ne cessent de réduire la ration de pain pendant les deux mois qui suivent ${ }^{58}$. Le témoignage de Tim McCarthy permet aussi de conclure à des brimades physiques. Les Allemands «s'acharnent sur les prisonniers ${ }^{59}$ », confisquent leurs bottes, les obligeant ainsi à travailler pieds nus dans la neige. L'éreintement physique s'accompagne alors d'un épuisement psychologique.

Alors qu'en temps de guerre les punitions collectives visent à «faire pression sur l'État adverse ${ }^{60} »$, les représailles à l'encontre des combattants irlandais ne cherchent nullement à faire pression sur les gouvernements ennemis. En aucun cas, les punitions ne s'inscrivent dans «un processus de négociation ${ }^{61}$ ». Les représailles du printemps 1917 contre les captifs français et britanniques envoyés travailler près des zones des combats cherchent, en ce sens, à protéger les prisonniers allemands gardés dans des conditions misérables $^{62}$. Dans le cas des captifs irlandais, il s'agit ici de briser toute loyauté envers la Couronne britannique. C'est sans compter le soutien moral du Père Crotty. Originaire de Kilkenny, et envoyé par le Pape en personne pour veiller aux besoins spirituels des prisonniers de guerre catholiques irlandais, l'aumônier dominicain apaise ses ouailles, les réconforte et les prie de ne pas commettre l'irréparable en leur rappelant leur serment d'allégeance envers la Grande-Bretagne ${ }^{63}$.

En février 1915, après plusieurs semaines de privations, les Allemands regroupent à nouveau les captifs irlandais, amaigris, faibles, affamés ${ }^{64}$. Après de sévères privations, les gardiens du camp les amènent par petits groupes dans le hall où se trouve " une large table recouverte de nourriture appétissante ${ }^{65}$ », table garnie « de viande rouge, de pommes de terre

\footnotetext{
54 TNA, WO 141/15, « Rapport sur les visites de Sir Casement au camp de Limburg, 1916 », témoignage de Thomas Fahey.

55 Ibidem, témoignage de Thomas Higgins.

${ }^{56}$ Ibidem, WO 161/101, Index 3/177, «Commission sur le traitement des prisonniers britanniques dans les camps allemands : rapports individuels de prisonniers ».

57 Ibidem., WO 161/98/140, «Commission sur le traitement des prisonniers britanniques dans les camps allemands, rapports individuels de prisonniers ».

58 Ibidem, WO 161/98/245, «Commission sur le traitement des prisonniers britanniques dans les camps allemands, rapports individuels de prisonniers ».

59 Ibidem, WO 161/99/864, «Commission sur le traitement des prisonniers britanniques dans les camps allemands, rapports individuels de prisonniers ».

${ }^{60}$ Stéphane Audoin-Rouzeau et Annette Becker, 14-18, retrouver la guerre, Paris, Folio histoire, 2000 , p. 122.

${ }^{61}$ Heather Jones, Violence against Prisonniers of War, op. cit., p. 129.

${ }^{62}$ Ibidem, pp. 161-162.

${ }^{63}$ TNA, WO 141/49, «Rapport sur le camp de prisonniers de Limburg et sur la tentative de formation d'une brigade irlandaise, $1921 »$, p. 4.

${ }^{64}$ Ibidem, WO 141/9, «Formation d'une brigade irlandaise parmi les prisonniers de guerre en Allemagne », témoignage du caporal Michael O’Connor, 12 novembre 1915.

65 Ibidem, WO 161/100/1131, «Commission sur le traitement des prisonniers britanniques dans les camps allemands, rapports individuels de prisonniers ».
} 
et de thé66 », afin de tester une nouvelle fois la capacité de résistance et la loyauté des soldats. Cette fois, ce sont les deux transfuges, Michael Keogh et Timothy Quinlisk, qui s'adressent à leurs anciens frères d'armes. Par petits groupes, les gardiens amènent les soldats pour un entretien avec les «officiers» de la brigade. En échange de leur engagement, les «transfuges » leur promettent une libre circulation à l'intérieur de la ville et une nourriture abondante. Ils leur certifient également que les autorités allemandes les laisseraient s'installer en Allemagne ${ }^{67}$ ou leur distribueraient des passeports pour rejoindre les États-Unis une fois la guerre finie ${ }^{68}$.

Les Irlandais se retrouvent face à un dilemme : combattre pour les Allemands et retrouver leur liberté, échappant ainsi aux privations de guerre, ou continuer de tenir. Les pressions qu'exercent les responsables du camp et les «transfuges » nouvellement ralliés au Kaiser fragilisent moralement les hommes. Écrasés, accablés par un univers limité et oppressant, les captifs sont tiraillés entre la trahison, le «transfert » ou le statu quo mortifère. Douze sont exécutés afin d'inciter d'autres captifs à s'engager ${ }^{69}$. Rien n'y fait; les combattants résistent. Les Allemands séparent alors les sous-officiers et les soldats ${ }^{70}$. Entre le 29 avril et le 29 mai 1915, une douzaine de prisonniers quitte précipitamment Limburg pour Lauban, Gorlitz, Crossen, Guben et Spottan ${ }^{71}$.

En mai 1915, après une nouvelle visite de Sir Casement et une politique d'épuisement menée depuis presque six mois, une cinquantaine de prisonniers «mourant de faim ${ }^{72}$ » renoncent à l'emprisonnement. Les hommes quittent le camp de Limburg, défaits de leur loyauté envers la Couronne britannique. Le caporal James Conaghan, du $2^{\text {ème }}$ bataillon des Royal Inniskilling Fusiliers, confie même que «des Anglais [et] des Ecossais ${ }^{73}$ » prêtent allégeance à Guillaume II. Un soldat anglais du $1^{\mathrm{er}}$ bataillon du Cheshire Regiment confirme aussi la présence de combattants anglais de l'armée régulière dans la brigade irlandaise ${ }^{74}$.

\section{RENIER L'UNIFORME BRITANNIQUE : LES « TRANSFUGES » DE LA BRIGADE IRLANDAISE}

Les nouveaux «transfuges» se heurtent à l'animosité de leurs frères d'armes. Le soldat écossais Patrick Cullen, du Corps Expéditionnaire britannique, n'hésite pas à tancer les «traîtres » sur leur passage. Les autres prisonniers les insultent. Les captifs britanniques extériorisent leur rancœur et affichent ouvertement leur loyauté envers la Couronne. L'indignation verbale s'apparente ici à un acte de résistance que les Allemands s'empressent de mater. Aussitôt deux sentinelles se ruent sur un prisonnier écossais, le frappant à la tête d'un coup de revolver. La brutalité du choc jette le soldat à terre ; il est ensuite roué de coups

\footnotetext{
${ }^{66}$ TNA, WO 161/99/864, «Commission sur le traitement des prisonniers britanniques dans les camps allemands, rapports individuels de prisonniers ».

67 Ibidem, WO 161/99/140, «Commission sur le traitement des prisonniers britanniques dans les camps allemands, rapports individuels de prisonniers ».

${ }^{68}$ Ibidem, WO 141/49, « Rapport sur le camp de prisonniers de Limburg et sur la tentative de formation d'une brigade irlandaise, $1921 »$, p. 7.

${ }^{69}$ Ibidem, WO 161/101, Index 3/2311 et 1793, « Commission sur le traitement des prisonniers britanniques dans les camps allemands : rapports individuels de prisonniers ».

${ }^{70}$ Ibidem, WO 141/49, « Rapport sur le camp de prisonniers de Limburg et sur la tentative de formation d'une brigade irlandaise, $1921 »$, p. 8.

${ }^{71}$ Ibidem, WO 141/15, «Rapport sur les visites de Sir Casement au camp de Limburg, 1916 », témoignage de Michael None.

72 Ibidem, WO 161/98/165, «Commission sur le traitement des prisonniers britanniques dans les camps allemands, rapports individuels de prisonniers ».

73 Ibidem, WO 161/99/972, «Commission sur le traitement des prisonniers britanniques dans les camps allemands, rapports individuels de prisonniers ».

${ }^{74}$ Ibidem, WO 161/101, Index 2/1998, «Commission sur le traitement des prisonniers britanniques dans les camps allemands : rapports individuels de prisonniers. »
} 
avant d'être mis au trou pendant trois jours. La trahison de soldats du Corps Expéditionnaire britannique suscite une colère générale. Les fidèles de l'Empire y voient une soumission impardonnable devant l'ennemi. Les Allemands prennent d'ailleurs soin d'évacuer discrètement les volontaires afin de leur éviter des représailles de la part de leurs anciens frères d'armes ${ }^{75}$.

Comment comprendre que seule une cinquantaine d'hommes accepte de renier l'uniforme britannique sur les quelque 2000 prisonniers qui se trouvent à Limburg ? Est-ce à dire que le renoncement à la liberté chez les autres induit une loyauté envers la Couronne britannique? À en croire un sergent de Galway, «s'il y avait une chance ne serait-ce qu'infime de victoire, les hommes seraient d'accord pour combattre pour l'Irlande ${ }^{76} »$. Or ils jugent l'entreprise vaine. D'autant plus qu'il n'y a aucune garantie de succès. Combattre avec l'armée britannique ne signifie donc pas forcément que les hommes juraient une loyauté inconditionnelle envers la Couronne. Quelques soldats irlandais réfléchissent à un possible « transfert de loyauté » mais se gardent de commettre l'irréparable.

Plusieurs captifs refusent de s'engager dans la brigade irlandaise afin de protéger leur famille. En effet, les femmes des soldats comptent sur les indemnités de séparation (separation allowances) pour subvenir aux besoins de leur famille. Le gouvernement britannique verse 5 shillings par semaine pour chaque femme ainsi qu'un complément pour chaque enfant du foyer ${ }^{77}$. Si des soldats acceptaient de prêter serment à Guillaume II et que le gouvernement britannique découvrait l'identité des «transfuges", les répercussions ne se feraient pas attendre. S'engager dans la brigade irlandaise, c'est aussi se couper du soutien de son pays. Les desseins allemands font l'objet d'une attention très particulière au RoyaumeUni. Des marraines de guerre cessent d'ailleurs de correspondre avec les captifs engagés dans la brigade irlandaise. Les «transfuges » reçoivent des lettres dans lesquelles les citoyennes s'insurgent contre leur choix et refusent de continuer à correspondre avec « les traîtres de Roger Casement ${ }^{78}$ ». Même de simples soupçons de passage à l'ennemi suffisent à priver un prisonnier de tout soutien matériel. Ainsi, les marraines de guerre de l'Association des femmes irlandaises de Kensington Palace cessent-elles immédiatement d'envoyer des colis de vivres à un soldat du $2^{\text {ème }}$ bataillon des Connaught Rangers soupçonné de trahison. La secrétaire de l'Association des Familles de Soldats et de Marins de Thurles se renseigne auprès du Ministère pour savoir si le soldat Patrick Keogh, matricule 10771, a effectivement rejoint la cause allemande ${ }^{79}$. Une citoyenne irlandaise d'Inver, dans le comté de Queenstown, supplie elle aussi le Ministère de lui indiquer si Michael O'Callaghan, du $2^{\text {ème }}$ bataillon des Royal Munster Fusiliers, sert désormais l'ennemi allemand. Depuis novembre 1915, cette marraine de guerre lui fait régulièrement parvenir des vivres mais refuse de continuer à correspondre avec lui s'il s'avère qu'il a effectivement rejoint la brigade irlandaise ${ }^{80}$. Grâce à la Commission Gouvernementale sur le Traitement des Prisonniers de Guerre Britanniques ${ }^{81}$, établie dès 1915, les autorités parviennent très vite à identifier les «transfuges ». En recoupant plusieurs témoignages de soldats et officiers échangés ou évadés des camps allemands, le Ministère de la Guerre finit par obtenir, en 1916, la liste complète de tous les

\footnotetext{
${ }^{75}$ NLI, MS 13.085/25, «Correspondance entre le Père Nicholson et Sir Casement », 15 mars 1915.

${ }^{76}$ Idem.

77 John Faries, "The Development in England of a State System for the Care of the Disabled Soldier", in Publications of the Red Cross Institute for Crippled and Disabled Men, New York, The Red Cross, 1918, p. 10.

${ }^{78}$ NLI, MS 13.085/1.iv, « Correspondance au sujet de la Brigade irlandaise », lettre de Keogh à Gaffney (?), 21 mai 1916.

${ }^{79}$ TNA, WO 141/9, «Formation d'une brigade irlandaise parmi les prisonniers de guerre en Allemagne », lettre de Mademoiselle Cooke au Ministère de la Guerre, 21 février 1916.

${ }^{80}$ Ibidem, lettre du lieutenant-colonel J. Barry au Ministère de la Guerre, 8 mars 1916.

${ }^{81}$ Ibidem, WO 161/98-136, «Commission sur le traitement des prisonniers britanniques dans les camps allemands, rapports individuels de prisonniers. »
} 
combattants de la brigade irlandaise. En ce sens, rejoindre la brigade irlandaise, ce n'est pas seulement se priver d'un réconfort moral mais aussi d'un soutien matériel. Au-delà des lettres réconfortantes de l'arrière, tout ce qui provient du pays permet de tenir. Les prisonniers comptent sur les marques d'attention de leur nation pour supporter la vie à l'intérieur du camp. À ce sujet, un sous-officier irlandais n'omettait d'ailleurs pas d'avertir ses hommes que les noms des traitres seraient vite connus des autorités britanniques ${ }^{82}$. Rien, si ce n'est la parole de Sir Casement et des autorités allemandes, ne garantissait de meilleures conditions de détention et la victoire allemande.

Ne parvenant pas à faire fléchir les hommes, le gouvernement allemand doit donc se contenter d'une «brigade irlandaise » insignifiante d'une cinquantaine de soldats. Conscients de l'échec de leur plan, les autorités se résignent et mettent immédiatement fin au traitement spécial des prisonniers irlandais ${ }^{83}$.

Le 17 juillet 1915, les officiers et soldats de la «brigade irlandaise » partent en train pour le camp de Zossen, à une trentaine de kilomètres au sud de Berlin, un camp où les Allemands regroupent tous les prisonniers non-européens d'origine africaine et asiatique afin de les inciter à renier l'uniforme sous lequel ils combattent. Afin de séduire les captifs musulmans, les autorités allemandes font « construire une mosquée et [leur] offrent toutes les facilités religieuses, au nom de l'alliance de l'Islam $^{84} »$.

À Zossen, les gardiens distribuent des laissez-passer pour les sergents qui peuvent visiter les abords du camp jusqu'à 18 heures accompagnés de cinq ou six hommes. Le général permet ainsi aux «officiers »d'obtenir des sorties permanentes jusqu'à 20 heures tous les soirs $^{85}$. Pourtant, les captifs vivent toujours dans des casernes militaires clôturées par des barbelés et surveillées en permanence par une sentinelle ${ }^{86}$. Les conditions de détention indignent les combattants de la brigade. Comment les Allemands peuvent-ils garder leurs alliés dans un camp gardé de fils de fer barbelés et d'une sentinelle ? La déception laisse bientôt place à un sentiment d'amertume ${ }^{87}$. Les laissez-passer qui permettent aux gradés irlandais de sortir du camp et de se rendre à l'auberge entre 19 et 20 heures les scandalisent. Les combattants de la brigade irlandaise ressentent la semi-liberté comme une insulte. La présence d'une sentinelle à l'entrée du camp fait douter les soldats irlandais de la sincérité des autorités allemandes. De juin 1915 à juin 1916, regroupés avec quelque 15000 Français, les combattants irlandais de Zossen considèrent que « la vie ressemble beaucoup à celle dans un camp de prisonniers ordinaire ${ }^{88} »$.

Les soldats irlandais échappent pourtant à ces baraques de bois et aux clôtures de fils de fer barbelés qui «nécrosent les espoirs les plus tenaces ${ }^{89}$ ». Les quelques moments de liberté que les gardiens concèdent aux transfuges leur permettent de voir autre chose que « les limites physiques du camp ${ }^{90} »$, source d'éreintement psychologique, de résignation, d'abrutissement.

\footnotetext{
${ }^{82}$ TNA, WO 141/49, «Rapport sur le camp de prisonniers de Limburg et sur la tentative de formation d'une brigade irlandaise, $1921 »$, p.1.

${ }^{83}$ NLI, MS 13.085/20, «Correspondance entre Sir Casement et le Père Crotty', lettre du Père Crotty à Roger Casement, 30 novembre 1915

${ }^{84}$ Annette Becker, Oubliés de la Grande Guerre, op. cit., p. 326.

${ }^{85}$ NLI, MS 13.085/17, «Correspondance de Roger Casement », lettre de Haln à Roger Casement, 24 octobre 1915, p. 3 .

${ }^{86}$ Ibidem, MS 13.085/21, «Correspondance entre Sir Casement, Keogh et Quinlisk », lettre de Keogh à Roger Casement, 4 septembre 1915.

${ }^{87}$ Ibidem, MS 13.085/1.ii, «Correspondance au sujet de la brigade irlandaise », lettre de Zerhusen à Roger Casement, 9 octobre 1915, p. 3.

${ }^{88}$ Ibidem, MS 13.085/1.ix, « Correspondance au sujet de la brigade irlandaise », lettre de Quinslik à Gaffney, 27 octobre 1915.

${ }^{89}$ Annette Becker, Oubliés de la Grande Guerre, op. cit., p. 91.

${ }^{90}$ Ibidem, p. 98.
} 
La très relative clémence des autorités allemandes se heurte vite à l'ingratitude des officiers irlandais qui les déçoivent et les agacent. À de nombreuses reprises, les frasques de certains sous-officiers ne manquent pas d'indigner les habitants de Zossen et de Danzig qui viennent par exemple se plaindre de l'attitude d'un certain Mahoney ivre mort sur la voie publique et voleur de denrées alimentaires ${ }^{91}$. Le commandant doit ordonner à plusieurs hommes de maitriser l'ivrogne ${ }^{92}$. En octobre 1915, le général interdit formellement à ses hommes d'offrir à boire aux soldats irlandais ${ }^{93}$.

À Zossen, les peines d'emprisonnement restent fréquentes. Une absence injustifiée du caporal Mahoney lui vaut une peine d'emprisonnement de trois jours. Le soldat de première classe Burke subit la même peine pour insubordination et refus de travail ${ }^{94}$. Les gardiens punissent de deux semaines d'enfermement le soldat Brandon. Mais leur statut varie, semblet-il, selon les circonstances. Lors du procès de quatre membres de la brigade irlandaise, le 20 octobre 1915, le tribunal condamne les prisonniers de guerre à quinze jours d'emprisonnement pour avoir volé de l'argent à des captifs algériens ${ }^{95}$. Le jugement stipule bien que les combattants de la brigade irlandaise ne sont, et n'ont jamais cessé d'être, des «prisonniers » ennemis. Au début de l'année 1916 le commandant de la brigade déplorait d'ailleurs que « tous [les] hommes à l'exception de deux sous-officiers se trouvent [à présent] internés et sous surveillance ${ }^{96} »$ avant d'ajouter : «ils ne sont rien moins que des prisonniers de guerre ${ }^{97} »$.

\section{LE DEPART DE ZOSSEN : CONSEQUENCE IMMEDIATE DU SOULEVEMENT DE 1916}

Le 24 avril 1916, une rébellion éclate au cœur de la capitale irlandaise. Une poignée de nationalistes investissent plusieurs quartiers du centre-ville et déclarent la guerre à l'Empire britannique. Les quelque 1700 rebelles défient les forces britanniques de l'île et les obligent à faire venir des renforts ${ }^{98}$. La brigade irlandaise du Kaiser ne participe pas au soulèvement. Les Allemands avaient refusé que les soldats irlandais quittent le territoire. Berlin s'était contenté d'envoyer à bord d'un paquebot battant pavillon norvégien, 20000 fusils, 10 mitrailleuses et 5 millions de munitions aux insurgés ${ }^{99}$. Sir Casement se trouvait à bord de 1'Aud mais les autorités britanniques interceptèrent le navire avant que les insurgés ne parviennent à décharger le convoi ${ }^{100}$.

D'Allemagne, les soldats de la brigade irlandaise assistent avec impuissance à l'échec du soulèvement. Cinq jours après l'éclatement de la révolte, les rebelles rendent les armes ${ }^{101}$. Alors que les autorités militaires exécutent les meneurs des Pâques Sanglantes, l'existence

\footnotetext{
${ }^{91}$ NLI, MS 13.085/21, «Correspondance entre Sir Casement, Keogh et Quinlisk », lettre de Keogh à Roger Casement, 7 septembre 1915.

${ }^{92}$ Ibidem, lettre de Keogh à Roger Casement, 24 octobre 1915.

${ }^{93}$ Ibidem, MS 13.085/1.ii, «Correspondance au sujet de la brigade irlandaise », lettre de Zerhusen à Roger Casement, 9 octobre 1915, p. 3.

${ }^{94}$ Ibidem, MS 13.085/17, «Correspondance de Roger Casement », lettre de H. Haln à Roger Casement, 24 octobre 1915, p. 2.

${ }^{95}$ Ibidem, MS 13.085/21, «Correspondance entre Sir Casement, Keogh et Quinlisk », lettre de Keogh à Roger Casement, 20 octobre 1915.

${ }^{96}$ Ibidem, MS 13.085/1.iii, «Correspondance au sujet de la brigade irlandaise », lettre de Robert Monteith au capitaine Nadoley, 5 février 1916.

${ }^{97}$ Idem.

${ }^{98}$ D.J. Hickey and J.E. Doherty, A Dictionary of Irish History, 1800-1980, Dublin, Gill \& Macmillan, 1987, p. 143.

${ }^{99}$ Royal Commission on the Rebellion in Ireland; Report of the Commission, June 26, 1916, p. 9.

${ }^{100}$ Max Caulfield, The Easter Rebellion, Dublin, Gill \& Macmillan Ltd, 1995, pp. 29-37.

${ }^{101}$ Ibidem, p. 277.
} 
même de la brigade irlandaise semble compromise. Le soulèvement avorté, les Allemands déplacent bientôt la brigade en Prusse occidentale. Le départ de la brigade irlandaise coïncide avec l'échec de l'insurrection.

En juin 1916, tous les soldats de la brigade irlandaise quittent le camp de Zossen pour Danzig, quartier général du $17^{\text {ème }}$ Corps de l'Armée allemande, près de la mer Baltique en Prusse occidentale. Les «transfuges » irlandais y gardent les quelque 16000 prisonniers russes qui s'y entassent tandis que d'autres partent travailler sans escorte dans les fermes des villages ${ }^{102}$. Le soir, sous la responsabilité de leurs sous-officiers, les hommes sortent du camp lors des promenades en ville. Là, il leur est possible de se procurer des vivres, de prendre un verre à la terrasse d'un café, de revivre un moment parmi les civils ${ }^{103}$.

Les transfuges obtiennent aussi la possibilité de partir se baigner. La veille du $1^{\mathrm{er}}$ juillet 1916, alors même que les combattants de la $36^{\text {ème }}$ division d'Ulster attendent que les gradés donnent l'assaut, recroquevillés sur la crosse de leur fusil, sous les tirs d'obus incessants qui creusent la terre ${ }^{104}$, dix-huit soldats de la brigade irlandaise partent pour un après-midi au bord de l'eau. Le 3 juillet, alors que les cadavres en décomposition des soldats d'Ulster gisent dans le No Man's Land et qu'à l'arrière les amputations se succèdent ${ }^{105}$, une quarantaine de soldats de la brigade partent à bord d'automobiles spécialement mises à disposition par la Kommandantur, pour une après-midi de baignade dans la mer Baltique ${ }^{106}$. Dans cette même période, les Allemands entament une campagne de représailles contre les captifs britanniques. Environ 2000 soldats britanniques quittent Döberitz pour la mer Baltique. Là, dans des conditions spartiates, les internés subissent des mesures de rétorsion, réponse au gouvernement britannique qui avait déplacé 1500 prisonniers allemands d'Angleterre vers Rouen et Le Havre en avril $1916^{107}$. Sur les territoires de la Russie occupée, par moins 10 degrés, les Allemands frappent à coups de crosse les Britanniques et leur infligent le supplice du poteau, punition qui consiste à attacher un homme et à le laisser suspendu par les bras. En août 1916, 253000 captifs travaillent près des zones de combat sur un total de 1625000 hommes faits prisonniers par les Allemands ${ }^{108}$.

Les transfuges irlandais échappent à ces expériences traumatisantes mais sont à présent tenus de travailler. Les Allemands constituent deux sections de travail de prisonniers irlandais : l'une à Stolp et l'autre à Dirschau. À Stolp, une douzaine de prisonniers travaillent dans une imprimerie, certains dans des fermes allemandes, tandis que d'autres trouvent une occupation comme celle de pompier sur un bateau à vapeur ${ }^{109}$. Les autorités allemandes étouffent sous les plaintes incessantes des Irlandais qui insistent pour «être payés plus qu'un ouvrier agricole allemand ${ }^{110} \gg$. Le salaire de ces travailleurs irlandais équivaut au salaire des prisonniers de guerre, à savoir 30 pfennigs par jour. C'est ce qui pousse un soldat à démissionner, arguant qu'il refusait d'être traité comme un « esclave de 5 heures du matin à 9

\footnotetext{
102 TNA, WO 141/49, «Rapport sur le camp de prisonniers de Limburg et sur la tentative de formation d'une brigade irlandaise, $1921 »$, p. 10.

${ }^{103}$ NLI, MS 13.085/1.iv, «Correspondance au sujet de la brigade irlandaise », lettre de Keogh à Gaffney (?), 21 mai 1916.

104 Archives de la Bibliothèque universitaire de Leeds, Peter Liddle Collection, GS 1039, James McRoberts, $14^{\mathrm{ème}}$ bataillon des Royal Irish Rifles, « Recollections, 1914-1918 », p. 1834.

105 Archives Publiques d'Irlande du Nord, D1507/A/18/2, «Description de l'attaque de la $36^{\text {ème }}$ division par le capitaine Wilfrid B. Spender, 2 juillet $1916 »$.

${ }_{106}$ NLI, MS 13.085/1.iv, «Correspondance au sujet de la brigade irlandaise », lettre de Zerhusen à Gaffney, 3 juillet 1916.

${ }^{107}$ Heather Jones, Violence against Prisoners of War, op. cit., p. 135-137.

108 Ibidem, p. 133.

${ }^{109}$ NLI, MS 13.085/1.v, « Correspondance au sujet de la brigade irlandaise », lettre de Hahn à Gaffney, 4 juillet 1916.

${ }^{110}$ Idem.
} 
heures du soir ${ }^{111} »$ pour si peu. Les Allemands demandent alors personnellement aux fermiers de rémunérer les soldats irlandais à hauteur de 75 pfennigs par jour afin de contenir leur mécontentement ${ }^{112}$. Mais les autorités s'impatientent devant l'ingratitude des Irlandais qui « refusent de travailler, quittent sans cesse le camp et reviennent quelques jours plus tard en braillant : 'cela ne me convient pas' ${ }^{113}$ ». Cette situation est d'autant plus incompréhensible que la Kommandantur multiplie les démarches auprès des fermiers allemands « qui disposent d'une main d'œuvre russe qu'ils peuvent sous-payer ${ }^{114}$ ». À Danzig les captifs semblent d'ailleurs multiplier les actes d'indiscipline ${ }^{115}$. Policiers et juges des villages avoisinants implorent les autorités du camp de renvoyer les Irlandais. Ces derniers ne peuvent s'empêcher de causer des problèmes. Vols, chapardages, passages à tabac de policiers ; les incidents se succèdent. Les patrons d'usines refusent désormais d'employer des prisonniers qui s'absentent et demandent l'avance de leur solde avant de disparaître.

Les privilèges dont jouissent les membres de la brigade irlandaise disparaissent peu à peu, si bien qu'à partir de juillet 1916, si ce n'est une relative liberté et une nourriture correcte, les «transfuges » redeviennent des captifs à part entière. Ils n'échappent pas au sort commun des prisonniers contraints de travailler dans des usines ou des entreprises agricoles, souvent bien éloignées des camps. Là encore, les Allemands prennent soin de ne pas leur assigner de travaux difficiles, tels le drainage de marécages. Mais désormais ils travaillent dans quelques-uns de ces 100000 camps de travail sortis de terre en Allemagne ${ }^{116}$.

\section{CONCLUSION}

Les combattants irlandais du Corps Expéditionnaire britannique capturés par les Allemands lors des premiers mois du conflit bénéficient au début de leur internement d'un traitement enviable. Séparés de leurs frères d'armes britanniques, les prisonniers profitent quelque temps de la relative clémence des autorités allemandes qui s'emploient à gagner leur confiance. Mais les captifs comprennent bientôt que derrière les privilèges se cache la volonté de les forcer à renier l'uniforme britannique. En ce sens, les relations historiques tendues entre l'Irlande et la Grande-Bretagne façonnent le regard que les autorités allemandes portent sur les combattants irlandais. Les accords conclus entre les rebelles irlandais et Berlin s'inscrivent dans la ligne de la politique étrangère allemande visant à déséquilibrer de l'intérieur les puissances de l'Entente ${ }^{117}$.

Que cherchaient véritablement les Allemands? Tout au plus, une «attaque de diversion dans l'espoir de soulever une rébellion ou un coup d'État contre la présence britannique $^{118}$ ». L'Irlande s'apparentait à une zone périphérique où des troubles auraient pu mettre en difficulté les Britanniques. Les autorités françaises, quelques heures à peine après l'éclatement du soulèvement, comprirent qu'il s'agissait là d'une stratégie visant à « empêcher [leurs] alliés d'envoyer en France les renforts destinés à augmenter les effectifs de

\footnotetext{
${ }^{111}$ NLI, MS 13.085/1.v, « Correspondance au sujet de la brigade irlandaise », lettre de Hahn à Gaffney, 4 juillet 1916.

112 Idem.

${ }^{113}$ Idem.

${ }^{114}$ Ibidem, lettre de Zerhusen à Gaffney, 5 août 1916.

115 Ibidem, MS 13.085/1.iv, «Correspondance au sujet de la brigade irlandaise », lettre de Zerhusen à Gaffney, 20 mai 1918.

${ }^{116}$ Annette Becker, Oubliés de la Grande Guerre, op. cit., p. 113.

${ }^{117}$ Maurice Carrez, «Première Guerre mondiale et identité nationale en Finlande », in Rémy Cazals, André Loez (dir.), 1914-1918, Identités troublées. Les appartenances sociales et nationales à l'épreuve de la guerre, Toulouse, Éditions Privat, 2011, p. 302.

118 Jérôme aan de Wiel, The Irish Factor, 1899-1919, op. cit., p. 22.
} 
l'armée anglaise ${ }^{119} »$. Sir Casement se rendit compte, lui aussi, que l'insurrection importait plus pour les Allemands que la levée d'une brigade irlandaise. «Nous n'avons aucune ambition réaliste en Irlande [...] si ce n'est une diversion militaire ${ }^{120} \gg$ lui expliquerait plus tard le chef de la section «politique»du grand état-major général allemand. Seule donc importait pour les Allemands une éventuelle rébellion sur le territoire irlandais capable de nuire aux intérêts britanniques.

119 Ministère des Affaires étrangères, la Courneuve, 1CPCOM545, Cabinet du Ministre, « Correspondance politique et commerciale, Guerre 1914-1918, Irlande », rapport du colonel de la Panouse, attaché militaire à l’Ambassade de la République française à Londres, à Monsieur le Ministre de la Guerre, 3 mai 1916.

${ }^{120}$ Jérôme aan de Wiel, The Irish Factor, 1899-1919, op. cit., p. 194. 
Emmanuel Destenay est chercheur associé au LabEx «Écrire une Histoire Nouvelle de l'Europe» (Université Paris I-Paris IV Sorbonne) et Visiting Fellow en Relations internationales à l'Université de Stanford. Il a soutenu sa thèse financée par l'Institut de Recherche Stratégique de l'École militaire de Paris (IRSEM) sur la participation des engagés volontaires irlandais pendant la Première Guerre mondiale à l'Université Paris IV-Sorbonne en 2014. Au-delà d'une étude strictement limitée à la période 1914-1918, ses recherches doctorales traitaient également de la sortie de guerre des anciens combattants irlandais et des répercussions de la guerre d'Indépendance (1919-1921) et de la guerre civile (1922-1923) sur leur réinsertion sociale et économique. En 2015, il a reçu le Prix de thèse de la Fondation irlandaise de France.

\section{RESUME}

Pendant la Première Guerre mondiale, les prisonniers de guerre irlandais bénéficient d'un traitement singulier. Le gouvernement allemand sépare les captifs irlandais des autres prisonniers britanniques quelques mois après le début des hostilités et les regroupe dans un seul et même camp. Nourriture abondante, conditions de détention enviables, possibilités de lire et de fumer; tout cela tranche singulièrement avec le sort des prisonniers de guerre ordinaires. Lorsque les hommes reçoivent la visite de Sir Casement, ancien diplomate britannique sensible à la cause nationaliste et fervent défenseur d'une indépendance irlandaise, les prisonniers comprennent que les Allemands espèrent les inciter à renier l'uniforme britannique. En cherchant à instrumentaliser les tensions historiques entre l'Irlande et la Grande-Bretagne, les Allemands réfléchissent au moyen de lever une brigade irlandaise qui combattrait contre l'armée britannique en Irlande. Les mauvais traitements que subissent ensuite les prisonniers s'inscrivent ici dans une volonté de les forcer à trahir la GrandeBretagne. Alors que les violences envers les prisonniers de guerre visent généralement à briser les résistances individuelles ou à faire pression sur les gouvernements ennemis, les punitions endurées par les captifs irlandais s'inscrivent ici dans un dessein visant à les inciter à renier l'uniforme britannique et à rallier la cause allemande.

\section{MOTS-CLES}

PREMIERE GUERRE MONDIALE, ALLEMAGNE, BRIGADE IRLANDAISE, PRISONNIERS DE GUERRE, PROPAGANDE DE GUERRE

\section{ABSTRACT NOTICE}

During the First World War, Irish prisoners of war enjoyed special treatment. A few months after the outbreak of war, the German government separated Irish captives from other British soldiers and housed them together in a single camp. They were given plenty to eat, enviable living conditions, reading material and tobacco in striking contrast to the usual treatment of prisoners of war. When the men had a visit from Sir Casement, former British diplomat, sympathetic to the nationalist cause and an ardent supporter of an independent Ireland, the POWs understood that the Germans expected them to reject their British uniform. In their quest to excite historic tensions between Ireland and Great Britain, the Germans imagined a way to raise an Irish force to overcome the British army in Ireland. The ill treatment to which the prisoners were afterwards subjected can be put down to the desire to force them to betray 
Great Britain. While aggression towards prisoners of war usually aimed to break down individual resistance or put pressure on enemy governments, the punishments and deprivation endured by the Irish captives were exacted in order to incite them to support the German cause. After the failure of the German government's strategy which succeeded in mobilising a mere fifty men, the events that took place in Ireland on the eve of this First World War conflict shaped the way that the enemy looked on Irish troops.

KEYWORDS

FIRST WORLD WAR, GERMANY, IRISH BRIGADE, PRISONERS OF WAR, WAR PROPAGANDA 